\title{
Structural Optimization of Shell Side of Condenser Based on Porous-Medium Model
}

\author{
Hongcai Wang ${ }^{1, \text { a }}$, Jinqiao $\mathrm{He}^{2, \mathrm{~b}}$ \\ ${ }^{1}$ School of Energy Science and Engineering,Central South University,Changsha 410083,China \\ ${ }^{2}$ Institute of Energy Source \& Power Engineering, Changsha University of Science \& \\ Technology, Changsha 410004, China \\ ahongcai_001@163.com, b274179626@qq.com
}

\begin{abstract}
Keywords: condenser; porous-medium; tube-bundle; structural optimization
Abstract. According to the characteristics of fractal and distributed resistance of the condenser tube-bundle area, a porous medium model with three kinds of porosity is established based on the steam exhaust flow in the tube-bundle, and the changes of kinetic pressure and flow of steam exhaust around the inlet of bundle under different load were analyzed. The corner results show that the pressure distribution of the upper steam space of the condenser shell side was affected by the position and form of outer inlet corner of the upper tube-bundle area, and it leads to larger kinetic pressure change with direct sharp-angled transition, and the kinetic pressure disturbance is significantly enhanced with the load increase. The inlet kinetic pressure of the upper tube-bundle area depends on the push of condenser's exhaust pressure and the lower area on the condensation effect. It can reduce the kinetic pressure by strengthening or densifying the upper tube-bundle arrangement of the lower tube-bundle area, but it is necessary to slow down the tendency of the flow in this area and avoid work medium draining. The design margin of the lower edge length of lower tube-bundle area is too large, while that of the upper edge length of upper tube-bundle area is too narrow for the inflowing steam exhaust to expand and condense in time in the corresponding tube-bundle area.
\end{abstract}

\section{Introduction}

The cold-junction of steam turbine refers to equipment and piping systems of an effective thermodynamic cycle of cold source in the last stage blade outlet of steam turbine $\rightarrow$ condenser $\rightarrow$ circulating water system whose main equipment include condenser, circulating pumps, vacuum pumps and cooling tower [1]. According to related researches, one more $1 \mathrm{kPa}$ of back-pressure of cold-junction of the steam turbine leads to a $0.7 \%$ to $0.9 \%$ [2] increase in heat consumption rate of corresponding generation. Characteristics of steam flow as well as condensation heat transfer of the shell side of condensers of steam turbines in power plant serve as an important factor in setting the back pressure of cold-junction steam turbine. With the development of high-power turbine unit and more complex condenser structure, the flow characteristics of condensation heat transfer of steam-side exhaust flow turns to be more complex [3-5], contributing to great distribution in the back pressure of steam turbine under different load, which will affect the entire thermal power units in terms of thermal economy and security $[1,6]$.

Porous-medium model takes tubes as a porous skeleton so as to make for easier mesh generation and significant reduction in the mesh quantity and computing load with an effective reflection of the flow characteristics of porous-medium during heat transfer [7,8]. The present numerical studies of shell and tube heat exchangers generally establish two or three dimensional numerical model with solutions for shell and tube heat exchangers by applying distributed resistance methods proposed by Patankar and Spalding [9-14]. China also sees a lot of exploratory work of numerical simulation in the application of porous medium model $[15,16]$. Given the heat transfer of tube-bundle in cross-flow for steam exhaust of shell and tube condensers, there is a big difference in the condenser tubes in different areas in terms of arrangement, which easily leads to uneven admission of steam exhaust flowing into tube-bundle area along the bundle. Therefore, an in-depth analysis of flow 
characteristics of circumferential inlet steam exhaust flow of tube-bundle area of tube and shell condensers is necessary for structural optimization of the tube-bundle arrangement of this condenser.

\section{Mathematical Model Analyses of Condensers in Thermal Power Plants}

An Analysis of Condenser Structure and Operating Characteristics.The surface, two-pass halving-style condensers of $310 \mathrm{MW}$ thermal power units in Zhuzhou Power Plant features single-back pressure, single shell side and condenser tube-bundles of transversal arrangement.

The steam exhaust of condenser flow along condenser pipes' longitudinal direction is of significant characteristics of condenser tube-bundles in cross-flow. For the small flow resistance of cooling water in the tube, non-susceptible to plugging, easy processing and scaling-up, this type of condenser has been widely applied. However, caused by complex piping arrangement, steam exhaust in the inner shell side leads to a great air distribution in the tube bundle, which will make back-pressure distribution in last-stage exhaust of steam turbine deteriorating the operating conditions of cold-junction condensing steam turbine unit.

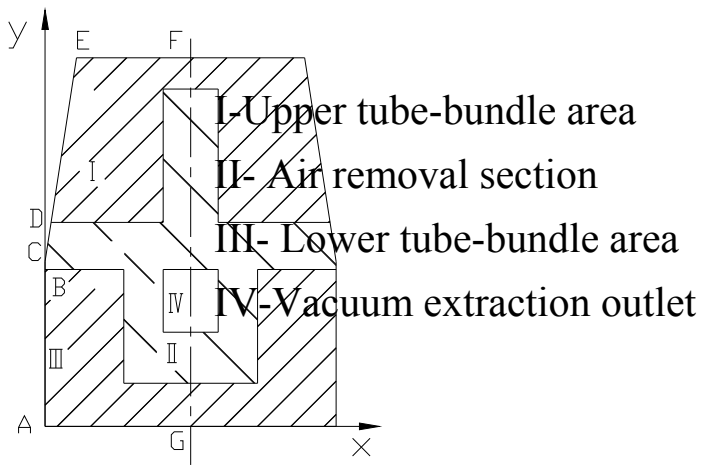

Fig.1 Condenser tube bundle arrangement of 310MW unit in Zhuzhou power plant

Fig. 1 is the tube-bundle arrangement diagram of this condenser tube-bundle. Because of the great structural difference in the different tube-bundles of condenser tube bundle with halving-type characteristics [9-13], and different porosities of tube-bundle parts, the tube-bundle area, in accordance with characteristics of porous-medium model, should be divided into three parts of different porosities, namely: upper tube-bundle area, lower bundle -tube area and air removal section, as it is shown in Table 1.

Table 1 The porosity of different tube-bundle areas

\begin{tabular}{ccc}
\hline Upper tube-bundle area & Air removal section & Lower tube-bundle area \\
\hline 0.5828 & 0.9795 & 0.5284 \\
\hline
\end{tabular}

With upper and lower tube-bundle areas' two-pass cooling effect of shell side's outside elbows connected, air removal section in tube-bundle middle area sees a significant gap between the upper and lower tube-bundle areas at the edge of tube bundles. And this condition would lead part of the steam exhaust flow directly into the air removal section. So, air removal section is also arranged with a number of cooling water pipes so as to further improve condensation efficiency of the condenser.

Heat Transfer Characteristics of Steam Exhaust of Tube-bundle Area.The condenser applies horizontal tube condensation, that is, driven by circulating pump, cooling water in the condenser continuously flows through the cooling pipes and steam exhaust is condensed in the outer wall of condensers. Since the steam exhaust temperature is not high, heat transfer coefficient of condensers is mainly affected by condenser water-side fouling, thickness of steam-side condensate film, pipe arrangement, air content in the steam and other factors, rather than the heat dissipation of condenser and outside environment. Obviously, condensate film thickness is mainly determined by the surface properties of the material, and regular cleaning of water-side fouling also can eliminate its impact on heat transfer, hereafter the main factors in different heat transfer performance in different positions of condensers' tube-bundle areas are piping arrangement and air content in the steam. For steam exhaust condensation, a small amount of non-condensable gases outside leaking into the condenser will see a 
relative concentration increase, which will lead to reduced partial pressure of steam exhaust, and thus steam exhaust finds it difficult to condense in the cooling pipe surface, exerting huge influence on condensation heat transfer process[2,12]. But non-condensable gas, for its small amount, normally impacts the heat exchange condensation of air removal section.

Analysis of Porous-Medium Model.Steam flow of condensers' shell sides is a multi-component complex process involving phase change seen inside and outside of cooling tube-bundles at the same time. A study on flow characteristics of the flow and heat transfer process in the condenser calls for simplification and assumes in the following aspects: ignoring the effects of temperature and liquid volume; sufficient pumping capacity and non-condensability of air pump; condensers' adiabatic shells and heat released from condensation all transferring to cooling water; ignoring the effects of tube sheets, support members and exhaust outlet tubes, etc. Flow in each steam chest may be approximated as two-dimensional steady flow of steam exhaust flowing horizontally through the bundle.

In addition, the condensers' working conditions is assumed that: steam condensation in saturation; cooling water flows in their cooling tubes maintain equal; ignoring the effect of non-condensable gas on heat transfer, air and steam in the condenser is uniformly mixed; equal pressure in tube-bundles of outer shell side.

Based on the above assumptions, factors influencing the last-stage of the turbine steam exhaust pressure mainly are about the condenser tube-bundle area. So by taking tube-bundle area as porous zone and in which the steam flowing as that in porous medium, a porous-medium model with distributed resistance and distributed mass sinks can be created, as follows.

Mass Continuity Equation:

$$
\frac{\partial(\varepsilon \rho \mathrm{u})}{\partial \mathrm{x}}+\frac{\partial(\varepsilon \rho \mathrm{v})}{\partial \mathrm{y}}=\mathrm{Q}
$$

X-direction Momentum Conservation Equation:

$$
\begin{aligned}
& \frac{\partial}{\partial \mathrm{x}}\left(\varepsilon \rho \mathrm{u}^{2}\right)+\frac{\partial}{\partial \mathrm{y}}(\varepsilon \rho \mathrm{uv})=\varepsilon \rho \mathrm{f}-\frac{\partial}{\partial \mathrm{x}}(\varepsilon \rho)+\frac{\partial}{\partial \mathrm{x}}\left[\varepsilon\left(\mu^{\prime}-\frac{2}{3} \mu \mathrm{e}\right) \mathrm{div} \overline{\mathrm{V}}\right] \\
& +\frac{\partial}{\partial \mathrm{x}}\left(2 \mu \mathrm{e} \varepsilon \frac{\partial \mathrm{u}}{\partial \mathrm{x}}\right)+\frac{\partial}{\partial \mathrm{y}}\left[\varepsilon \mu \mathrm{e}\left(\frac{\partial \mathrm{v}}{\partial \mathrm{x}}+\frac{\partial \mathrm{u}}{\partial \mathrm{y}}\right)\right]+\mathrm{Q} \mathrm{u}
\end{aligned}
$$

Y-direction Momentum Conservation Equation:

$$
\begin{aligned}
& \frac{\partial}{\partial \mathrm{x}}(\varepsilon \rho \mathrm{uv})+\frac{\partial}{\partial \mathrm{y}}\left(\varepsilon \rho \mathrm{v}^{2}\right)=\varepsilon \rho \mathrm{y}-\frac{\partial}{\partial \mathrm{y}}(\varepsilon \rho)+\frac{\partial}{\partial \mathrm{y}}\left[\varepsilon\left(\mu^{\prime}-\frac{2}{3} \mu_{\mathrm{e}}\right) \operatorname{div} \overline{\mathrm{V}}\right] \\
& +\frac{\partial}{\partial \mathrm{y}}\left(2 \mu_{\mathrm{e}} \varepsilon \frac{\partial \mathrm{v}}{\partial \mathrm{y}}\right)+\frac{\partial}{\partial \mathrm{x}}\left[\varepsilon \mu_{\mathrm{e}}\left(\frac{\partial \mathrm{v}}{\partial \mathrm{x}}+\frac{\partial \mathrm{u}}{\partial \mathrm{y}}\right)\right]+\mathrm{Q}_{\mathrm{v}}
\end{aligned}
$$

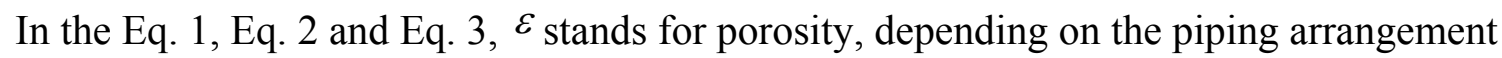
properties of tube-bundle area; $\mu^{\prime}$ for inertial resistance coefficient, $\mu_{\mathrm{e}}$ for equivalent viscosity coefficient, $\mathrm{f}_{\mathrm{x}}, \mathrm{f}_{\mathrm{y}}$ for two friction factors between the cooling tube-bundles, both depending on the load characteristics of condensers, and $\mathrm{Q}, \mathrm{Q}_{\mathrm{u}}, \mathrm{Q}_{\mathrm{v}}$ for the source terms.

\section{Flow Characteristics Analysis of Inlet Steam Exhaust of Tube-Bundle Area}

As shown in Fig. 1, most steam exhaust sees heat transfer condensation by going through DE section as well as EF section and its axisymmetric side into the upper tube-bundle area; through $\mathrm{AB}$ section as well as AG section and its axisymmetric side into the air removal section; and through $\mathrm{BC}$ section as well as CD section and its axisymmetric side into the air removal section. There are no distinctive internal boundaries between the three air removal sections. The differences in number of condenser tubes and structure of condenser tubes' arrangement result in steam exhaust flowing in the three air removal sections and this effect is ultimately reflected by steam exhaust pressure and flow distribution of tube-bundle's outer inlet.

Inlet Flow Characteristics of Upper Tube-Bundle Area. Fig.2 demonstrates the kinetic pressure and flow distribution of steam exhaust around D, E and F, inlets of tube-bundle area. Fig.2 (a) shows 
that, as load increases, kinetic pressure mounts at an increasing growth rate with a significant increase in the inlet around the corner. Kinetic pressures around the inclined inlet of EF section and horizontal inlet of DE section are substantially equal. With a sudden increase along EF section, kinetic pressure reaches a maximum at point $\mathrm{E}$, then decreases along the $\mathrm{X}$-direction, and maintains stable from $0.7 \mathrm{~m}$ point. Thus, it's recognized that the pressure about the upper steam space of the condenser shell side is distributed by arrangement position and form of outer steam inlet at the corner of tube bundle area. Sharp-angled transition leads to a greater change of kinetic pressure, and kinetic pressure disturbance is largely strengthened with the load increased, which will significantly affects the stream flow characteristics of condenser's upper tube-bundle area.
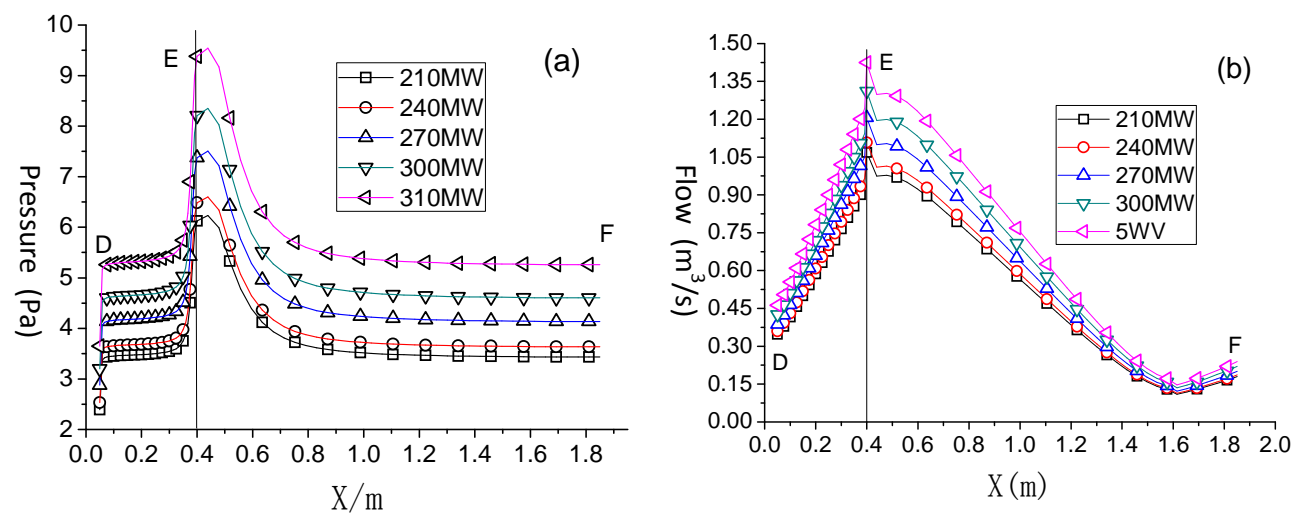

$\mathrm{a}$ - inlet kinetic pressure distribution $\mathrm{b}$ - inlet flow distribution

Fig.2 Inlet kinetic pressure and flow distribution at D, E and F

Fig.2 (b) shows that, flow at the corner of point E reaches the maximum, and then decreases at the minimum and bottoms out from the $1.5 \mathrm{~m}$ point to point $\mathrm{E}$ in the $\mathrm{EF}$ section. And at $\mathrm{E}$, the flow sees a big difference in values with different loads. Therefore, upper inlet of the upper tube-bundle area should have three recirculation zones, which is detrimental to the condensation of upper tube-bundle and easily leads to the uneven steam exhaust flow in shell side's non- tube-bundle area.

Inlet Flow Characteristics of Air Removal Section.Fig.3 is about kinetic pressure and flow distribution of steam exhaust at B, C and D, inlets of air removal section. Fig.3 (a) shows that kinetic pressure remains unchanged in the inlet section, and grows with the increase of the load and sees a rapid increase of kinetic pressure from the $0.05 \mathrm{~m}$ point to two ends, due to condensation of upper and lower tube-bundles. Theoretically, the greater the kinetic pressure at both ends, the more difficultly the steam exhaust flows into air removal section, thus reducing steam exhaust directly discharged through vacuum extraction outlet of air removal section. But given the two-pass design formed by import and export water tank by diaphragms between the tube bundles, there must be a gap between the upper and lower tube bundles. In this context, the gap width and the arrangement of upper and lower tube-bundle areas will affect the amount of direct discharged steam exhaust.
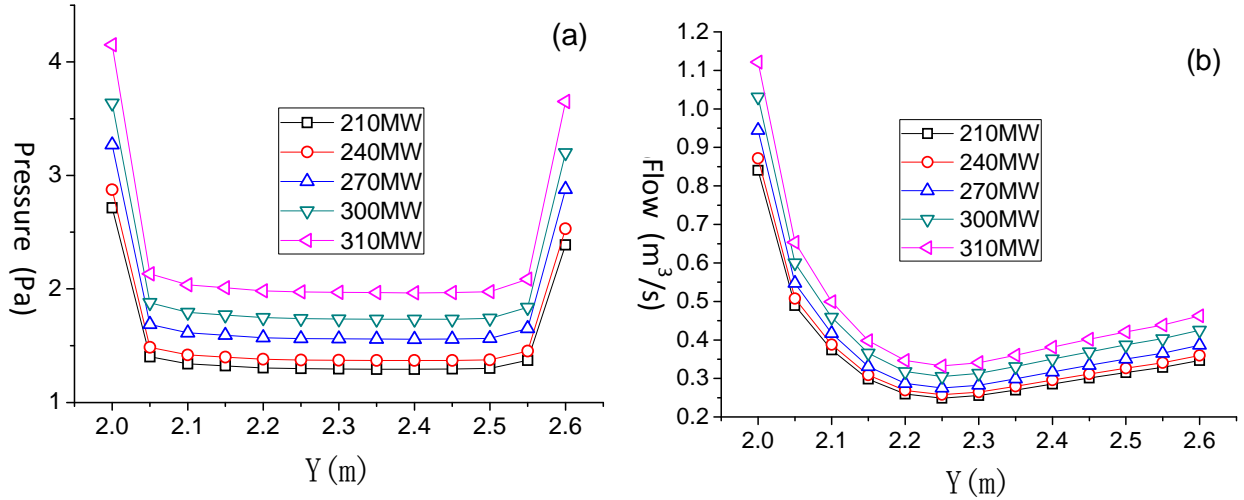

$\mathrm{a}$ - inlet kinetic pressure distribution $\mathrm{b}$ - inlet flow distribution

Fig. 3 Inlet kinetic pressure and flow distribution of air removal section

According to Fig.3 (b), inlet section flow of air removal section reduces along the B, C and D direction and bottoms out slowly at its minimum of $2.25 \mathrm{~m}$ height, while the vacuum extraction outlet 
of lowest pressure is arranged in air removal section surrounded by lower tube-bundle. Along the vacuum extraction outlet is arranged with tube-bundles circumferentially, but it is necessary to strengthen the arrangement of upper tube-bundles of lower bundle-tube area.

Inlet Flow Characteristics of Lower Tube-bundle Area. Fig.4 is about kinetic pressure and flow distribution of steam exhaust of inlets along AB and AG sections, lower tube-bundle area. Fig.4 (a) and (b) shows that, in addition to a larger kinetic pressure area in the corner A, the kinetic pressure of steam exhaust around inlets $\mathrm{AG}$ and $\mathrm{AB}$ of lower tube-bundle area is almost equal. Since B point in $\mathrm{AB}$ section serves as the boundary point between air removal section and lower tube-bundle area, it faces less pressure, because of less condenser pipes in air removal section and its bad cooling effect, and the corresponding kinetic pressure is also reduced. And different from kinetic pressure distribution at the corner $\mathrm{E}$ in the upper bundle area, its counterpart along the inlets, $\mathrm{AB}$ and $\mathrm{AG}$ sections, is equally distributed with distributions ranging within $0.3 \mathrm{~m}$ to point $\mathrm{A}$. Its trend and size also sees a symmetrical distribution with as symmetrical point.
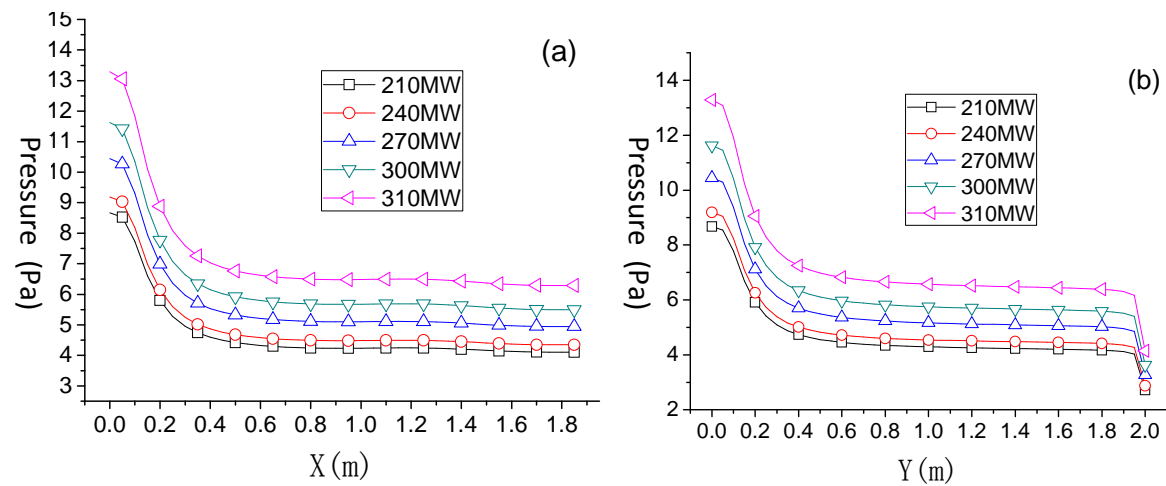

(b)
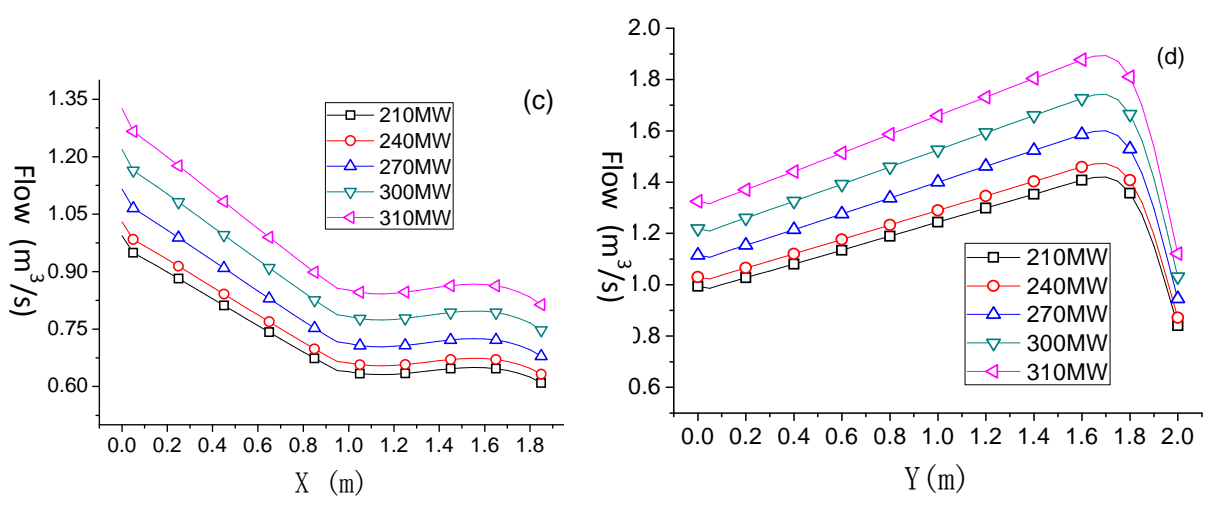

a- kinetic pressure distribution of $A G$ inlet $b$ - kinetic pressure distribution of $A B$ inlet c - flow distribution of AG inlet

$\mathrm{d}$ - flow distribution of $\mathrm{AB}$ inlet

Fig.4 Inlet kinetic pressure and flow distribution of lower tube-bundle area

Fig.4 (c) shows that the flow distribution of steam exhaust flowing into the lower tube-bundle area along the AG section and along the EF section of upper tube-bundle both experience distributions, which is caused by the recirculation zones in the upper and lower tube-bundle areas. But relevant researches show that, on one hand, there is a flow increase at the intermediate point $F$ on the upper edge of the inlet of upper tube-bundle area, but on the other, steam exhaust flow of the inlet on the lower edge of the lower tube-bundle area is reduced along the AG section direction, then maintains at a stable value at $1 \mathrm{~m}$ before a slight increase, and continues to decrease at $1.65 \mathrm{~m}$ to the minimum at point G. As a consequent, it's seen that the design margin of lower edge length of lower tube-bundle area is too large, while the design width of the upper edge length of upper tube-bundle area is too narrow for the inflowing steam exhaust to expand and condense in time in the corresponding tube-bundle area.

As it's shown in 4 (d), the inlet flow of steam exhaust at the edge of $A B$ section reaches the maximum at $1.8 \mathrm{~m}$ from point $\mathrm{A}$, and it's reduced more rapidly near air removal section. According to Fig.2 (b) and 3 (b), the inlet flow near the lower tube-bundle side in air removal section also decreases 
rapidly along Y-direction, and fast bottoms out at the minimum of $2.25 \mathrm{~m}$ along Y-direction in the DE inlet section reaching the maximum at point $\mathrm{E}$ in upper tube-bundle area. Thus, there's a big difference in terms of fluid dynamics mechanism between the lateral inlet of upper and lower bundle areas, with the upper one depending on condensation effects and lower one mainly on the push of condenser's exhaust pressure.

\section{Conclusions}

The pressure distribution of the upper steam space of the condenser shell side is mainly affected by the position and form of outer inlet corner of the upper tube-bundle area. Sharp-angled transition leads to a greater change in kinetic pressure, and the kinetic pressure disturbance is significantly enhanced with the load increase.

There is a big difference in terms of flow kinetic mechanism between the lateral inlets of upper and lower bundle areas: the upper tube-bundle area depends mainly on the push of condenser exhaust pressure, while the lower one depends on the condensation effect. Densifying or strengthening arrangement of upper tube-bundle of lower tube-bundle area will lower the kinetic pressure, but which is necessary to slow down the tendency of the flow in this area and avoid uncondensed work medium draining.

Recirculation occurs around upper and lower inlets of the entire tube-bundle area, and which leads most increased flow caused by the load increases to flow into the two lateral inlets of tube-bundle area. And uneven condensation of tube-bundle area is more significant with increased load, which indicts that the design margin of lower edge length of lower tube-bundle area is too large, while the design width of the upper edge length of upper tube-bundle area is too narrow for the inflowing steam exhaust to expand and condense in time in the corresponding tube-bundle area.

\section{Acknowledgements}

This work was financially supported by the Project (2009CB219803-03) supported by the National Program on Key Basic Research Project (973 Program).

\section{References}

[1] Fangyou Xu, et al: Thermal Power Generation, Vol.31, No.7(2010), p.7-10 (in Chinese)

[2] Xuedong Wang, et al: Turbine Technology, Vol.52, No.4(2010), p.150-154 (in Chinese)

[3] Lanxin Zhou, et al: Proceedings of the CSEE, Vol.28, No.23(2008), p. 25-30

[4] Q.Y. Zhu, Y. Li: Int. J. Heat Mass Transfer, Vol.53, No. 7 (2010) , p.1417-1425

[5] Maozheng Yu, et al: Power Engineering, Vol.15, No.6(1995), p.42-49 (in Chinese)

[6] Zhuocheng Zhang.Large Power Station Condense, Beijing: China Machine Press,1993:4-5.

[7] Yanrui Li, et al: Applied Science and Technology, Vol.2 ( 2014) ,p.54-58 (in Chinese)

[8] Baorong Jia, et al:Proceedings of the CSEE, Vol. 8( 2009), p.14-19.

[9] Spalding D B:ICHMT Semonar, Dubrovnil, Vol.6(1967),p.1-15

[10] Patankar.S.V, Spalding.D B:Forsch, Ingenieurwes, Vol.4 (1978),p.4

[11] Q.Y. Zhu, et a: Int. J. Heat Mass Transfer, Vol. 53, No.8 (2010), p.3914-3927

[12] Jianlong Kou, et al:Journal of Applied Physics, Vol. 5 (2009),p.106

[13] Y. Ono, H. Mayama, et al:J. Colloid Interf. Sci, Vol.336(2009), p.215-225

[14] J.S. Wu, B.M. Yu:Int. J. Heat Mass Transfer, Vol.50, No.8( 2007), p.3925-3932

[15] Q.Y.Zhu, M.H.Xie, J.Yang, et al:Int. J. Heat Mass Transfer, Vol.54, No.7(2011), p.1400-1409

[16] Jinqiao He, Bo Xiao, et al:Journal of Central South University:Science and Technology, Vol.5 ( 2013), p.2113-2117 (in Chinese) 